$\mathrm{DTP} / 95 / 16$

January, 1995

\title{
New functional dilogarithm identities and sine-Gordon Y-systems
}

\author{
R.Tateo \\ Dipartimento di Fisica Teorica dell'Università di Toring冈 \\ via P.Giuria 1, I-10125 Torino, Italy \\ and \\ Department of Mathematics, University of Durhami \\ South Road, DH1 3LE Durham, England
}

\begin{abstract}
The sine-Gordon Y-systems and those of the minimal $M_{p, q}+\phi_{13}$ models are determined in a compact form and a correspondence between the rational numbers and a new infinite family of multi-parameter functional equations for the Rogers dilogarithm is pointed out. The relation between the TBA-duality and the massless RG fluxes in the minimal models recently conjectured is briefly discussed.
\end{abstract}

*e-mail: tateo@to.infn.it

†e-mail: roberto.tateo@durham.ac.uk 


\section{Introduction}

In [1] Al.B. Zamolodchikov has proposed a transformation of the TBA equations [2, 3, 4] for the so-called $A D E T$ diagonal scattering showing clearly their relation with a system of functional equations now known as $A D E T$ Y-systems. Many other Y-systems have now been discovered, and the importance of these relations in connection to the theory of integrable models has greatly increased: not only excited states, different phases of the same model and models that differ only in the boundary conditions imposed satisfy the same Y-system equations in all known cases, but also many definitely different models lead to the same equations [5, 6, 0, 8, 9]. The origin of this kind of universality is partially due to the rigid structure of this object, imposed by two remarkable properties: the stationary dilogarithm sum-rules, related to the UV and IR Casimir effect, and the periodicity related to the conformal dimension of the UV (IR) perturbing operator. In a recent paper 110 a new important mathematical property has been pointed out: using as a basic object the so-called $A D E T \diamond A D E T$ Y-systems [12, 13] a new family of multi-parameter functional dilogarithm 凹equations (FDE) [14 generalization of the Abel's and Euler's formulas has been proposed [2. The above two properties and the physical interpretation of this result given in [10] suggest the possibility of finding new FDE using the Y-systems of the known models: the aim of this letter is to show how to construct in a simple way FDE using the sine-Gordon TBA or its reduced form associated to the minimal models. The first part of this letter is devoted to the explicit formulation of these two families of $\mathrm{Y}$-systems in a compact form; this is also an original result and the "fusion at the massive node" [17] can be generalized to these Y-systems for obtaining the TBA equations of all the $Z_{n}$ supersymmetric versions [18] of the sine-Gordon or their reduced models. In the second part we present the result concerning the new functional dilogarithm identities and finally the relation between the TBA-duality connecting the theories $M_{p, q} \leftrightarrow M_{q-p, q}, Z_{p-1} \leftrightarrow M_{p, p+1}$ and the presence of non-unitary (unitary) massless fluxes in the minimal models is briefly discussed.

\footnotetext{
${ }^{1}$ In connection with the K-theory, R. de Jeu [15] has also checked that some of these Rogers's FDE have a corresponding $\mathrm{m}=2$ Bloch-Wigner [1] functional equation.

${ }^{2}$ See [16] for a list of the different branches of mathematics and physics in witch the dilogarithm emerging.
} 


\section{The sine-Gordon Y-systems}

The SG theory provides the simplest example of an exactly solvable relativistic quantum field theory. The SG Lagrangian is

$$
L=\frac{1}{2} \partial_{\mu} \phi \partial^{\mu} \phi+\frac{g \sqrt{4 \pi}}{\beta} \cos \left(\frac{\beta}{\sqrt{4 \pi}} \phi\right) \quad,
$$

and its S-matrix is the minimal $O(2)$-symmetrical solution of the unitarity, crossing and factorization equations [19]. The spectrum of the theory consists of a soliton and a number of soliton-antisoliton bound states with masses

$$
\begin{gathered}
M_{j}=2 M_{\text {sol }} \sin \left(\frac{j \xi}{2} \pi\right) \quad j=1,2, \ldots<\frac{1}{\xi}, \\
\xi=\frac{\beta^{2} / 8 \pi}{1-\beta^{2} / 8 \pi} .
\end{gathered}
$$

In order to obtain the TBA equations we have to apply the algebraic Bethe ansatz, and in developing this procedure in general in addition to the physical particles a finite number of pseudoparticles (also known as magnons) describing the colour interchange of the soliton must be introduced. The thermodynamic problem for the sine-Gordon model in the repulsive region is directly related to those of the XXZ models solved by Takahashi and Suzuki [20]. For finding the TBA equation in both the attractive and repulsive regimes directly from the SG S matrices we used standard methods [2, 5, 20, 21] and the details of this derivation are not important in this context. The passing from the TBA equation to a "minimal" compact expression for the associated Y-system is quite a direct but a tedious calculation, our results confirm the structure depicted in [22]: the sine-Gordon Y-system can be represented as a nested structure of a concatenation of $A_{n_{i}}$ Dynkins diagram ending with a $D_{n_{F}}$ diagram, to give a $D_{n_{T}}$-like Dynkin diagram. To be more precise, the Ysystem is uniquely defined by the simple continued-fraction 1 representation for the spectral parameter $\xi$ 团. Let us consider first the case $n_{1} \neq 0$,

$$
\xi=\frac{p}{q-p}=\hat{\xi}\left(n_{1}, n_{2}, \ldots, n_{F}\right):=\frac{1}{n_{1}+\frac{1}{n_{2}+\ldots \frac{1}{n_{F}-1}}},
$$

\footnotetext{
${ }^{3}$ See [23] for a rigorous definition and for a list of mathematical applications of the continued-fractions theory.

4 See [20] for a classification of the string solutions of the XXZ model using the simple continued-fractions.
} 
the TBA equation at this point contains $n_{1}$ breathers, a soliton and $\sum_{i=2}^{F} n_{i}$ magnons for a total of $n_{T}=\sum_{i=1}^{F} n_{i}$ (pseudo)particles . Defining the shifts

$$
s_{1}=\imath \pi \frac{\xi_{1}}{2} \quad, \quad s_{2}=\imath \pi \frac{\xi_{1} \xi_{2}}{2} \quad, \quad \ldots \quad, \quad s_{F}=\imath \pi \frac{\xi_{1} \xi_{2} \ldots \xi_{F}}{2} \equiv \frac{\imath \pi}{2 q-2 p}
$$

with

$$
\xi_{i}=\hat{\xi}\left(n_{i}, n_{i+1}, \ldots, n_{F}\right) \quad,
$$

we associate to any (pseudo)particle a node in a $D_{n_{T}}$ Dynkin-like diagram. In figure 1 a graphical representation, a generalization of those in [22 for the $\mathrm{SG}$ at $\xi=\frac{2 n+1}{2}$, is introduced for the readers convenience; the first $n_{1}$-nodes on the tail correspond to the breathers and the node $n_{1}+1$ to the soliton, the others are the magnons. In the following we shall indicate as $f \equiv n_{T}$ and $\bar{f} \equiv n_{T}-1$ the two nodes on the bifurcation of the $D_{n_{T}}$ diagram. Defining for any node a shift

$$
\mathcal{S}_{i}=s_{a} \quad \sum_{k=1}^{a-1} n_{k}<i \leq \sum_{j=k}^{a} n_{k}
$$

and for any pair of nodes $\{i, j\}$ an exponent

$$
c_{i, j}=c_{j, i}=(-1)^{a-1} \quad, \quad \sum_{k=1}^{a-1} n_{k}<i, j \leq 1+\sum_{k=1}^{a} n_{k},
$$

with $c_{i, j}=0$ if $i$ and $j$ are not adjacent. The Y-system for the nodes in the set $\left\{n_{1}, n_{1}+n_{2}, \ldots, n_{T}-n_{F}-n_{F-1}\right\}$, that is $k=\sum_{i=1}^{a} n_{i}$ and $a<F-1$, is

$$
\begin{aligned}
& Y_{k}\left(\theta+\mathcal{S}_{k}\right) Y_{k}\left(\theta-\mathcal{S}_{k}\right)=\left(1+Y_{k-1}(\theta)^{c_{k, k-1}}\right)^{c_{k, k-1}} \\
& \left(1+Y_{k+n_{a+1}+1}(\theta)^{\tilde{c}_{k}}\right)^{\tilde{c}_{k}} \\
& \begin{array}{c}
\prod_{j=k+1}^{k+n_{a+1}}\left(1+Y_{j}\left(\theta+\left(k+n_{a+1}-j\right) \mathcal{S}_{j}+\mathcal{S}_{k+n_{a+1}+1}\right)^{\tilde{c}_{k}}\right)^{\tilde{c}_{k}} \\
\prod_{j=k+1}^{k+n_{a+1}}\left(1+Y_{j}\left(\theta-\left(k+n_{a+1}-j\right) \mathcal{S}_{j}-\mathcal{S}_{k+n_{a+1}+1}\right)^{\tilde{c}_{k}}\right)^{\tilde{c}_{k}},
\end{array}
\end{aligned}
$$

with $\tilde{c}_{j}=c_{j, j+1}$. 

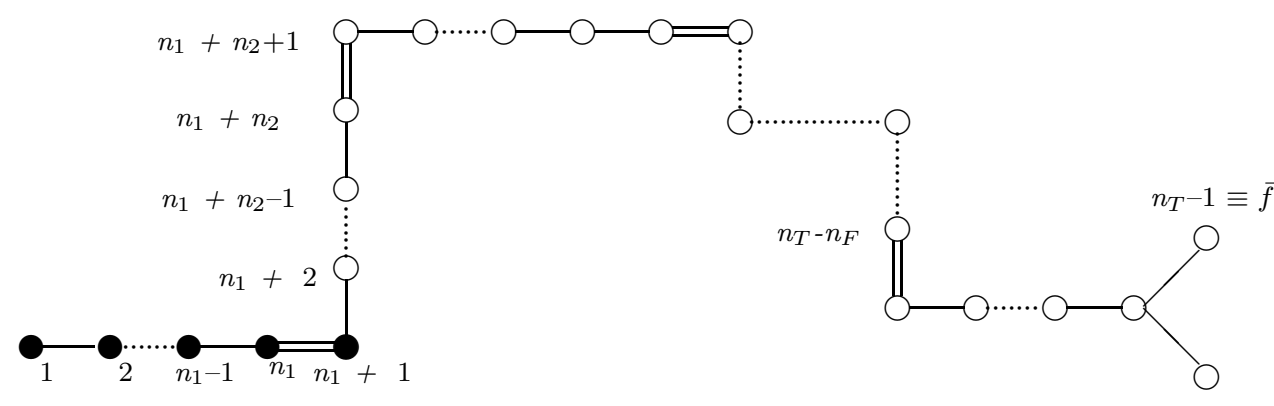

Figure 1: The TBA graph associated to the SG model in a rational point: there are $n_{1}$ black nodes associated to the breathers, the node $n_{1}$ corresponds to the soliton, all the other nodes are magnons. The quantity $c_{i, j}$ associated to a pair of nodes $\{i, j\}$ is equal to 0 if the nodes are not directly connected by a link, to 1 if the link is horizontal and -1 if vertical. The links on the bifurcation are horizontal if $F$ is even or vertical if $F$ is odd. The double-link defines the concatenation of an horizontal (vertical) with a vertical (horizontal) sub-diagram with a change of the shift $S_{i}$.

For the node $k=n_{T}-n_{F}$

$$
\begin{gathered}
Y_{k}\left(\theta+\mathcal{S}_{k}\right) Y_{k}\left(\theta-\mathcal{S}_{k}\right)=\left(1+Y_{k-1}(\theta)^{c_{k, k-1}}\right)^{c_{k, k-1}} \\
\left(1+Y_{f}(\theta)^{\tilde{c}_{k}}\right)^{\tilde{c}_{k}}\left(1+Y_{\bar{f}}(\theta)^{\tilde{c}_{k}}\right)^{\tilde{c}_{k}} \\
\prod_{j=k+1}^{n_{T}-2}\left(1+Y_{j}\left(\theta+\left(n_{T}-1-j\right) \mathcal{S}_{j}\right)^{\tilde{c}_{k}}\right)^{\tilde{c}_{k}} \\
\prod_{j=k+1}^{n_{T}-2}\left(1+Y_{j}\left(\theta-\left(n_{T}-1-j\right) \mathcal{S}_{j}\right)^{\tilde{c}_{k}}\right)^{\tilde{c}_{k}}
\end{gathered}
$$

for all the other nodes

$$
Y_{i}\left(\theta+\mathcal{S}_{i}\right) Y_{i}\left(\theta-\mathcal{S}_{i}\right)=\prod_{j \in a d j}\left(1+Y_{j}^{c_{j, i}}(\theta)\right)^{c_{j, i}} .
$$

In eq.(2.11) the sum runs over all adjacent nodes of the $D_{n_{T}}$ Dynkin diagram. Finally the Y-systems at $\bar{\xi}=1 / \xi$ in the repulsive region can be obtained from those at $\xi$ by the change $c_{i, j} \rightarrow-c_{i, j}$ and $s_{i} \rightarrow s_{i} / \xi$; this is the so-called TBA-duality in the sine-Gordon model. 
The reduced Y-system can be obtained as limit f f

$$
Y_{f}(\theta) \rightarrow-1 \quad, \quad Y_{\bar{f}}(\theta) \rightarrow-1 \quad,
$$

of the above equations, this also forces $Y_{n_{T}-2} \rightarrow 0$ or $Y_{n_{T}-2} \rightarrow \infty$. In taking the limit (2.12) we substitute the equation for the node $n_{T}-2$ in the equation for the node $n_{T}-n_{F}$. Considering that now the last three nodes decouple, equations (2.9,2.11) continue to hold. The only equation that formally changes is that for the node $k=n_{T}-n_{F}$

$$
\begin{gathered}
Y_{k}\left(\theta+\mathcal{S}_{k}\right) Y_{k}\left(\theta-\mathcal{S}_{k}\right)=\left(1+Y_{k-1}(\theta)^{c_{k, k-1}}\right)^{c_{k, k-1}} \\
\left(1+Y_{n_{T}-3}(\theta)^{-\tilde{c}_{k}}\right)^{-\tilde{c}_{k}} \\
\prod_{j=k+1}^{n_{T}-3}\left(1+Y_{j}\left(\theta+\left(n_{T}-1-j\right) \mathcal{S}_{j}\right)^{\tilde{c}_{k}}\right)^{\tilde{c}_{k}} \\
\prod_{j=k+1}^{n_{T}-3}\left(1+Y_{j}\left(\theta-\left(n_{T}-1-j\right) \mathcal{S}_{j}\right)^{\tilde{c}_{k}}\right)^{\tilde{c}_{k}}
\end{gathered}
$$

using the procedure of [12, 17, 22] the resulting Y-system can be used to describe all the minimal reduced theories of the $Z_{n}$ supersymmetric-sineGordon models, for instance the case $n=2$ corresponds to the minimal $N=1$ supersymmetric series. As usual the full TBA equations can be obtained via standard Fourier transformations.

\section{New functional dilogarithm equations}

The Rogers dilogarithm [14 $L(x)$ with $0 \leq x \leq 1$ is the unique function defined by the integral representation

$$
L(x)=-\frac{1}{2} \int_{0}^{x} d y\left[\frac{\log (y)}{1-y}+\frac{\log (1-y)}{y}\right],
$$

it is three times differentiable and satisfies the Euler functional equation

$$
L(x)+L(1-x)=L(1) \quad,
$$

5 The steps involved in the deduction of this result are formally the same described in 24. 
as well the five term relationship known also as the Spence and Abel functional equation

$$
L(x)+L(1-x y)+L(y)+L\left(\frac{1-y}{1-x y}\right)+L\left(\frac{1-x}{1-x y}\right)=3 L(1)
$$

Together with the periodicity properties that can be numerically verified with high precision

$$
\begin{aligned}
& Y_{i}\left(\theta+\imath \pi \frac{1+\xi}{2}\right)=Y_{i}(\theta) \\
& Y_{f}\left(\theta+\imath \pi \frac{1+\xi}{2}\right)=Y_{\bar{f}}(\theta) \\
& Y_{\bar{f}}\left(\theta+\imath \pi \frac{1+\xi}{2}\right)=Y_{f}(\theta)
\end{aligned}
$$

we have all the basic ingredients in order to construct our FDE. Following [10] we introduce the function

$$
H(x)=L\left(\frac{x}{1+x}\right) / L(1) \quad .
$$

and defining the quantities

$$
Y_{i}\left(\theta+\imath \pi \frac{m}{2 q-2 p}\right)=\Upsilon_{i}(m)
$$

with an appropriate number of initial conditions we find the following identities 9

$$
\mathcal{D}_{p, q}(S G)::=\sum_{i=1}^{n_{T}} \sum_{m=0}^{2 q-1} H\left(\Upsilon_{i}(m)\right)=2 q\left(\sum_{i=1}^{[(F+1) / 2]} n_{2 i-1}+(-1)^{F}\right)
$$

for the SG TBA. F is the integer defined in eq. (2.4) and $[(F+1) / 2]$ is the integer part of $(F+1) / 2$. For the RSG

$$
\mathcal{D}_{p, q}(R S G)::=\sum_{i=1}^{n_{T}} \sum_{m=0}^{2 q-1} H\left(\Upsilon_{i}(m)\right)=\mathcal{N}
$$

\footnotetext{
${ }^{6} \xi<1$ is implicit and the equations for $\bar{\xi}>\frac{1}{\xi}$ can be obtained by using the Euler formula.
} 
and

$$
\mathcal{N}=2 q\left(\sum_{i=1}^{F} 6 \frac{(-1)^{i+1}}{p_{i} q_{i}}+\sum_{i=1}^{[(F+1) / 2]} n_{2 i-1}-\left(\frac{3}{2}-(-1)^{F} \frac{7}{2}\right)\right)
$$

with $p_{1}=p, q_{1}=q$ but

$$
\frac{p_{i}}{q_{i}-p_{i}}=\hat{\xi}\left(n_{i}-1, n_{i+1}, \ldots, n_{F}\right) \quad,
$$

for $i>1$. The quantity $\mathcal{N}$ defined in eq. (3.22) is a positive integer and in the first simplest cases it takes the following values

$$
\mathcal{N}=2\left(n_{F}-3\right)\left(n_{F}-2\right)
$$

for $F=1$,

$$
\mathcal{N}=2\left(2 n_{F}-8 n_{1}+3 n_{F} n_{1}-n_{1}^{2}+n_{F} n_{1}^{2}-6\right)
$$

for $F=2$, and

$$
\begin{gathered}
\mathcal{N}=2\left(6-5 n_{F}+n_{F}{ }^{2}-5 n_{1}+2 n_{F} n_{1}+n_{1}{ }^{2}+11 n_{2}\right. \\
-6 n_{F} n_{2}+n_{F}{ }^{2} n_{2}+10 n_{1} n_{2}-5 n_{F} n_{1} n_{2} \\
\left.+n_{F}{ }^{2} n_{1} n_{2}-n_{1}{ }^{2} n_{2}+n_{F} n_{2} n_{1}{ }^{2}\right)
\end{gathered}
$$

for $F=3$. Equations (3.20) and (3.21) are the main result of this letter. In general in the sums (3.20,3.21 ) there are two identical independent FDE. It is possible to remove one solution with an identical choice of the initial conditions and dividing by two the final result. In the following we will assume that this procedure is applied. We have the following identification with the theories in [10]: $\mathcal{D}_{1, p}(S G) \equiv \mathcal{D}\left(D_{p} \diamond A_{1}\right), \mathcal{D}_{1, p}(R S G) \equiv \mathcal{D}\left(A_{p-3} \diamond A_{1}\right)$ and $\mathcal{D}_{2,2 p+3}(R S G) \equiv \mathcal{D}\left(T_{p} \diamond A_{1}\right)$, the first low rank new equation is the $\mathcal{D}_{3,7}(R S G)$ with four free parameters. The functional equations are

$$
\begin{gathered}
\Upsilon(m+3) \Upsilon(m-3)=(1+\mathcal{Z}(m+2))(1+\mathcal{Z}(m-2))\left(1+\frac{1}{\mathcal{Z}(m)}\right)^{-1} \\
\mathcal{Z}(m+1) \mathcal{Z}(m-1)=(1+\Upsilon(m))
\end{gathered}
$$


the with initial conditions $\{\Upsilon(0)=x, \Upsilon(2)=y, \Upsilon(4)=z, \mathcal{Z}(1)=k\}$ we find

$$
\begin{gathered}
\mathcal{D}_{3,7}(R S G) \quad:=H(k)+H(x)+H(y)+H(z)+ \\
H\left(\frac{1+y}{k}\right)+H\left(\frac{k(1+z)}{1+y}\right)+H\left(\frac{1+x}{k}\right)+ \\
H\left(\frac{(1+y)\left(1+2 k+k^{2}+x+k x+y+k y+x y+k z+k^{2} z\right)}{k x(1+k+y)(1+z)}\right)+ \\
H\left(\frac{(1+k)(1+x)(1+k+y)(1+z)}{y\left(1+2 k+k^{2}+x+k x+y+k y+x y+k z+k^{2} z\right)}\right)+ \\
H\left(\frac{(1+k)(1+k+y+k z)}{x(1+k+y)}\right)+H\left(\frac{1+k+x+k x+y+k y+x y}{k x y}\right)+ \\
H\left(\frac{1+k+y+z+k z+k y+k+k^{2} z}{y z}\right)+H\left(\frac{(1+k+x)(1+k+y)}{k z(1+k)}\right)=9
\end{gathered}
$$

\section{Comments and conclusions}

Finally let us make some comments on the TBA-duality characterized in 22 and connecting two theories in different regimes in the SG and in the RSG models. First we note that this duality-like relation is the off-critical version of the symmetry

$$
\frac{p}{q} \leftrightarrow \frac{q-p}{q}
$$

of the counting solution for the XXZ chain [30], and that in the RSOS models this transformation connect the critical line in the regime III/IV to that in the regime I/II. An important generalization of the L-state RSOS are the RSOS(P,P) models obtained by the fusion of $P \times P$ blocks of face weights together. The continuum limits of these theories in the regime III/IV are ( see ref. 32 and references therein) the $S U(2)_{P} \times S U(2)_{L-1-P} / S U(2)_{L-1}$ GKO constructions, but in the regime I/II all these theories have as same continuum limit the $Z_{L-1}$ models. This multi-to-one correspondence has an 
off-critical analogue: the $A_{1} \diamond A_{L-2}$ Y-systems describe all the perturbations $S U(2)_{P} \times S U(2)_{L-1-P} / S U(2)_{L-1}+\phi_{a d j}^{i d, i d}$, but the $A_{L-2} \diamond A_{1}$ describe only the $Z_{L-1}+\varepsilon_{1}$ model. It is not completely clear to us if this multi-to-one correspondence is also verified in the general case $L-P=\frac{p}{q-p}-1$. Obviously in this case instead of the $Z_{L-1}$ the common theory should be the theory $M_{q-p, q}$. Another interesting point consists in the existence of integrable perturbations connecting the theories in these two regimes; in 25 non perturbative fluxes between the $Z_{N}$ models and the unitary series have been proposed

$$
Z_{L-1}+\psi_{1} \bar{\psi}_{1}+\psi_{1}^{\dagger} \bar{\psi}_{1}^{\dagger} \rightarrow M_{L, L+1}+\phi_{3,1}+\text { higher order terms } .
$$

More recently in [28] also integrable perturbations in the non-unitary models generated by the operator $\phi_{2,1}$ have been conjectured; their generalizations imply a RG structure of the kind

$$
M_{p, q}+\phi_{2,1} \rightarrow M_{q-p, q}+\phi_{1,5}+\text { higher order terms , }
$$

so exactly connecting the $(P=1)$ RSOS models related by the TBA-duality! Naively speaking we also note that in at least two cases we can find fluxes connecting theories in one regime with theories in the other. The first example is the $Z_{4}$ model, there exist a massless unitary trajectory into the $M_{5,6}$ model of the kind (4.30), but also a second possibility exists, that is, perturbing the theory with a imaginary coupling constant [26]: in this case due to the "equivalence" [27] with the sine-Gordon model at $\xi=2$ we find as IR limit the theory $S U(2)_{2} \times S U(2)_{2} / S U(2)_{4}$. The simplest non unitary theory with at least two particles is the models $M_{2,7}$, in this case the TBA-duality fixes the correspondence

$$
M_{2,7} \leftrightarrow\left\{M_{5,7} \quad, \quad M_{7,12}\right\} \quad,
$$

the massless perturbations connecting the theory $M_{5,7}$ versus the theory $M_{2,7}$ is a consequence of the conjecture (4.31). The flow connecting the theory $M_{7,12}+\phi_{1,4}$ with the theory $M_{2,7}+T \bar{T}+$ h.o.t. can be found in the family of TBA of [13 and associated to the $T_{2} \diamond A_{2}$ theory. It is an interesting open problem to find other examples of this kind of non-perturbative RG fluxes in the RSOS(P,P) models. Finally we note that a generalization of the two-graphs tensorial product introduced in [29], should permit to find the TBA systems for many models in the families $\frac{G_{k} \times G_{l}}{G_{k+l}}$ with $k$ and $l$ particular rational numbers. 
Acknowledgements - We are greatly indebted to P.Dorey , F.Gliozzi and F.Ravanini for a lot of very useful discussion and help. We also are grateful to R. de Jeu and K.E.Thompson for useful discussions and the Mathematics Department of Durham University for the kind hospitality.

\section{References}

[1] Al.B.Zamolodchikov, Phys. Lett. B253 (1991) 391

[2] C.N. Yang and C.P. Yang, J. Math. Phys. 10 (1969) 1115

[3] Al.B.Zamolodchikov, Nucl. Phys. B342 (1990) 695

[4] T.Klassen, E.Melzer, Nucl. Phys. B338 (1990) 485

T.Klassen, E.Melzer, Nucl. Phys. B350 (1991) 635

[5] Al.B.Zamolodchikov, Nucl. Phys. B358 (1991) 497

Al.B.Zamolodchikov, Nucl. Phys. B358 (1991) 524

Al.B.Zamolodchikov, Nucl. Phys. B366 (1991) 122

[6] M.J.Martins Phys. Lett. B257 (1991)

[7] T.Klassen, E.Melzer, Nucl. Phys. B370 (1992) 511

[8] P.Fendley, Nucl. Phys. B374 (1992) 667

[9] V.V. Bazhanov, S.L. Lukyanov, Al. B. Zamolodchikov , CLNS 94/1316 hep-th/9412229

[10] F.Gliozzi , R.Tateo, Phys. Lett. B348 (1995) 84

[11] Don Zagier, Polylogarithms, Dedekind zeta functions, and the algebraic $K$-Theory of fields, Progress in mathematics.

[12] F.Ravanini, R.Tateo, A.Valleriani, Int. J. Mod. Phys. A8 (1993) 1707

[13] F.Ravanini, R.Tateo, A.Valleriani, Phys. Lett. B293 (1992) 361

E.Quattrini, F.Ravanini, R.Tateo, Bologna preprint DFUB 93-11

[14] L.Lewin, Dilogarithms and associated functions (MacDonald, 1958) 
[15] R. de Jeu private communications

[16] A.N.Kirillov,Dilogarithm identities hep-th/9408113

[17] P.Fendley , K.Intriligator, Nucl. Phys. B372 (1992) 533

[18] D.Bernard and A.Leclair, Phys. Lett. B247 (1990) 309

[19] A.B.Zamolodchikov, Al.B Zamolodchikov, Ann.Phys. (NY) 120 (1979) 253

[20] M.Takahashi, M.Suzuki, Prog.of Theo. Phys. 48 (1972) 2187

[21] M.D. Johnson, M. Fowler, Phys. Rev. B31 (1983) 536

[22] R.Tateo, Int. J. Mod. Phys. A10 (1995)

[23] L.Lorentzen, H.Waadeland, Continued fractions with applications Studies in computational Mathematics 3 , North-Holland

[24] P.Fendley , H.Saleur Nucl. Phys. B388 (1992) 609

[25] V.A.Fateev, Al.B.Zamolodchikov, Phys. Lett. B271 (1991) 91

[26] P.Fendley, H.Saleur, Al.B.Zamolodchikov, Int. J. Mod. Phys. A8 (1993) 5717

P.Fendley, H.Saleur, Al.B.Zamolodchikov, Int. J. Mod. Phys. A8 (1993) 5751

[27] V.A. Fateev, Int. J. Mod. Phys. A6 (1991) 2109-2132

[28] F.Ravanini, M.Staniskov, R.Tateo DFTT-31/94 hep-th/9411085

[29] F.Ravanini, Phys. Lett. B282 (1992) 73

[30] A. Berkovich , B.M. McCoy, Bonn-th-94-28 hep-th/9412030

[31] A.Klumper, P.A.Pearce, Physica A183 (1992) 304

[32] V.V. Bazhanov, N.Yu. Reshetikhin, Int. J. Mod. Phys. A4 (1989) 115 